Radiologe 2011 $51: 567$

DOI 10.1007/s00117-011-2201-3

Online publiziert: 19. Juni 2011

(c) Springer-Verlag 2011

S. Delorme $\cdot$ H.-P. Schlemmer Abt. E010-Radiologie, Deutsches Krebsforschungszentrum (DKFZ), Heidelberg

\title{
Bildgebung von Tumoren des weiblichen Beckens - eine interdisziplinäre Aufgabe
}

Liebe Leserinnen, liebe Leser,

die bildgebende Diagnostik von Tumoren im weiblichen Becken ist zunächst eine Domäne der transvaginalen Sonographie. Deren Auflösung ist unübertroffen; ihre Limitation liegt in der begrenzten Eindringtiefe. Gleichwohl, dies wird im Artikel von Prömpeler in diesem Heft zu Recht betont, sollte sich die Primärund Differenzialdiagnostik im Regelfall auf den Ultraschall beschränken. Neben einem System von „Referenzschallern“, wie es die DEGUM beispielhaft umgesetzt hat, das die Möglichkeiten des Ultraschalls auch auszuschöpfen hilft, benötigen wir einen engeren Dialog zwischen Gynäkologen und Radiologen, damit wir sowohl das Potenzial als auch die Grenzen der eigenen wie auch der Methode der anderen Disziplin besser verstehen. Nur so können wir zugleich überflüssige Diagnostik vermeiden und rechtzeitig abklärende Maßnahmen einleiten, wo es Not tut. Diesem Dialog ist das vorliegende interdisziplinäre Heft gewidmet.

Die eigentlichen Stärken von CT und MRT sind das Staging und die Verlaufskontrolle, sobald ein maligner Tumor gesichert oder sehr wahrscheinlich ist. State of the Art in diesem Feld ist Gegenstand der radiologischen Beiträge von Forstner, Colettini, Engelhard und Müller-Lisse. Aber die Radiologie hat noch mehr zu bieten: Neben der Embolisation steht für die Behandlung von Uterusmyomen der HIFU zur Verfügung. Eckey berichtet über dessen Einsatz unter MR-Monitoring der Therapieeffekte. Womit wir wieder beim Ultraschall wären...
Ihre

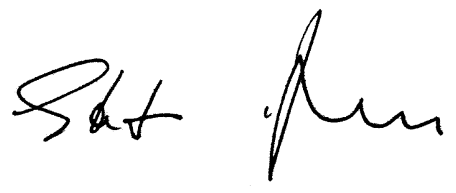

Prof. Dr. Stefan Delorme

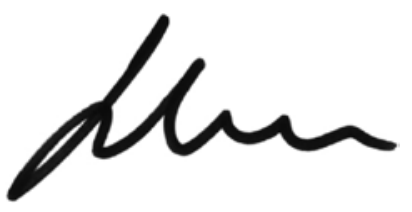

Prof. Dr. Heinz-Peter Schlemmer 